\title{
Thermodynamic Cyclic Voltammograms: Peak Positions and Shapes
}

\author{
Nicolas Georg Hörmann \\ Chair of Theoretical Chemistry and Catalysis Research Center, Technische Universität \\ München, 85748 Garching, Germany \\ Fritz-Haber-Institut der Max-Planck-Gesellschaft, Faradayweg 4-6, 14195 Berlin, Germany \\ E-mail: hoermann@fhi-berlin.mpg.de
}

\section{Karsten Reuter}

Fritz-Haber-Institut der Max-Planck-Gesellschaft, Faradayweg 4-6, 14195 Berlin, Germany

\begin{abstract}
Based on a mean-field description of thermodynamic cyclic voltammograms (CVs), we analyse here in full generality, how $\mathrm{CV}$ peak positions and shapes are related to the underlying interface energetics, in particular when also including electrostatic double layer (DL) effects. We show in particular, how non-Nernstian behaviour is related to capacitive DL charging, and how this relates to common adsorbate-centered interpretations such as a changed adsorption energetics due to dipole-field interactions and the electrosorption valency - the number of exchanged electrons upon electrosorption per adsorbate. Using $\operatorname{Ag}(111)$ in halidecontaining solutions as test case, we demonstrate that DL effects can introduce peak shifts that are already explained by rationalizing the interaction of isolated adsorbates with the interfacial fields, while alterations of the peak shape are mainly driven by the coverage-dependence of the adsorbate dipoles. In addition, we analyse in detail how changing the experimental conditions such as the ion concentrations in the solvent but also of the background electrolyte can affect the $\mathrm{CV}$ peaks via their impact on the potential drop in the DL and the DL capacitance, respectively. These results suggest new routes to analyse experimental CVs and use of those for a detailed assessment of the accuracy of atomistic models of electrified interfaces e.g. with and without explicitly treated interfacial solvent and/or approximate implicit solvent models.
\end{abstract}




\section{Introduction}

Cyclic voltammetry is a standard experimental technique for studying electrochemical interfaces that allows to infer surface compositions and interface reactions as a function of the applied electrode potential. In practice, cyclic voltammograms (CVs) are obtained by varying the electrode potential at fixed scan rate and measuring the current response of the electrode immersed in electrolyte solution. In general, CVs are characterized by potential regions within the stability window of the solvent which exhibit peaks of varying shape and height, and regions at low and high potentials, where faradaic, electrocatalytic reactions lead to exponentially increasing currents, e.g. due to the decomposition of the solvent. At high scan rates and/or in regions with faradaic reactions, CVs are governed by kinetic processes which typically induce a pronounced asymmetry for the forward and backward scan direction and/or a strong scan-rate dependence. Evidently, any theoretical description of such CVs necessitates the use of kinetic models [1], including potentially also macroscopic mass transport [2].

On the other hand, at low scan rates and in potential windows without faradaic side reactions this asymmetry typically vanishes as does the scan-rate dependence (when currents are appropriately normalized). In this case, $\mathrm{CV}$ peak positions and shapes are related directly to the underlying thermodynamics of the electrified interface [3], and such CV experiments provide invaluable contributions to the understanding of the latter. In turn, such CVs can be understood and simulated based on equilibrium thermodynamic considerations [4-12]. For such thermodynamic CVs the variation of the applied potential induces changes in the equilibrium surface charges, which can be traced back to changes in adsorbate coverages, as well as changes in the double layer charge, leading in sum to the observed electric current. As recently demonstrated for $\operatorname{Ag}(111)$ in halide containing solutions [13], double layer (DL) charging does not only add capacitive currents, but also affects equilibrium adsorbate coverages and the number of exchanged electrons per adsorbate - as expressed by the electrosorption valency [13-[16].

In this work we therefore analyse in most general terms thermodynamic CVs with included DL response. The derived equations can naturally explain Non-Nernstian behaviour and introduce a sensitive dependence of CV peaks to the electrolyte, via its impact on the interfacial capacitance [17, 18]. We hope, these results might help in the future to better understand according experiments and thus also help to validate and improve theoretical models. This is in particular important as at present all (atomistic) theoretical models of electrified interfaces necessarily introduce approximations due to computational time constraints, e.g in the complexity of the atomistic description (e.g. the surface with or without explicit water [19-23]), the quality of the energetics (e.g. empirical potentials [24]), the application of the electrode potential (e.g. absent or not [25-27], with explicit ions [28, 29] or an implicit solvent model [16, 22, 30-51]) or the derivation of macroscopic quantities (e.g. sampling [6, 10, 28, 52, 56], mean field models [9, 13]).

Here, we start from a fully grand canonical, ab initio thermodynamics description of the interface and derive in a consistent way expressions for the grand canonical meanfield energetics that includes effects of the potential up to second order. This second order 
approximation will be referred to as the $\mathrm{CHE}+\mathrm{DL}$ approximation as the terms up to linear order in the applied potential are identical to the terms frequently used to estimate the effects of the potential based on ab initio calculations at the potential of zero charge (PZC) and which is often referred to as the Computational Hydrogen Electrode [19, 57] (CHE) method. The quadratic, higher order term, on the other hand, captures capacitive DL charging with an assumed potential-independent interfacial capacitance, which was already successfully applied in many other works [13, 16, 37, 46, 54, 58, 63].

Naturally, the derived equations can be extended in a straightforward way to include any higher order response to the potential (e.g. of the DL capacitance), however, as we see later, any such energetics can not be treated analytically any more. At variance, the described $\mathrm{CHE}+\mathrm{DL}$ level of theory is largely amenable to rigorous mathematical analysis, and yields straightforward expressions for thermodynamic CVs. These results in combination with additional approximations, allow us to derive proxies for the position and the shape of peaks in according CVs that derive from electrosorption, which we then validate against the full theoretical description, and experiments. We demonstrate that both, peak positions and shapes, can be affected in a non-trivial way by the ion concentrations in solution as well as the electrolyte composition, and estimate according effects for halide solutions and selected metallic electrodes based on theoretical density functional theory (DFT) calculations.

\section{Theory}

\subsection{Ab initio thermodynamics of electrochemical interfaces}

In this work we concentrate for clarity on the description of metallic electrodes with just a single possible adsorbate species a (generalization to multiple species is conceptually straightforward). In this case, the Gibbs excess energy [13, 16, 50, 64, 65] at applied electrode potential $\Phi_{E}$ is defined as

$$
G_{\mathrm{exc}}^{\alpha}=G_{\mathrm{surf}}^{\alpha}\left(N_{\mathrm{s}}^{\alpha}, N_{\mathrm{a}}^{\alpha}, N_{e}^{\mathrm{abs}, \alpha}\right)-N_{\mathrm{s}}^{\alpha} \mu_{\mathrm{s}}-N_{\mathrm{a}}^{\alpha} \tilde{\mu}_{\mathrm{a}}+N_{e}^{\mathrm{abs}, \alpha} e \Phi_{E} .
$$

$\alpha$ denotes a certain interface configuration as characterized by its surface geometry (e.g. position of adsorbates) and chemical composition of $N_{\mathrm{s}}^{\alpha}$ substrate atoms, $N_{\mathrm{a}}^{\alpha}$ adsorbates of type a, and $N_{e}^{\mathrm{abs}, \alpha}$ electrons in excess to the charge-neutral pristine electrode surface. Expression (1) in essence determines the free energy cost of creating the interfacial system $\alpha$ with total energy $G_{\text {surf }}^{\alpha}\left(N_{\mathrm{s}}^{\alpha}, N_{\mathrm{a}}^{\alpha}, N_{e}^{\mathrm{abs}, \alpha}\right)$ when its constituents are taken from the thermodynamic reservoirs characterized by the (electro-)chemical potentials $\mu_{\mathrm{s}}, \tilde{\mu}_{\mathrm{a}}$ and $-e \Phi_{E}$ for substrate atoms, adsorbates and electrons, respectively.

With the adsorbates a present in solution as dissolved ions $\mathrm{A}^{\mathrm{q}_{\mathrm{a}} / \mathrm{e}}(\mathrm{aq})$ of charge $q_{\mathrm{a}}$, their electrochemical potential $\tilde{\mu}_{\mathrm{a}}$ can be determined from tabulated experimental equilibrium potentials $\Phi_{\mathrm{a}, \mathrm{eq}}^{\exp }$ of the redox reaction $\mathrm{A} \rightleftharpoons \mathrm{A}^{q_{\mathrm{a}} / e}(\mathrm{aq})+\frac{q_{\mathrm{a}}}{e} e^{-}(\mathrm{m})$ in combination with theoretical calculations of the chemical potential $\mu_{\mathrm{A}}$ in the reference phase. For hydrogen and halides, e.g., $\mu_{\mathrm{A}}$ is conventionally determined as half the chemical potential of the biatomic molecules at $298 \mathrm{~K}$ and 1 bar $\left(\mu_{\mathrm{A}}=\frac{1}{2} \mu_{\mathrm{A}_{2}(\mathrm{~g})}\right)$ and $\Phi_{\mathrm{a}, \text { eq }}^{\exp }$ refers to a $1 \mathrm{M}$ solution [66, 67]. In this case:

$$
\tilde{\mu}_{\mathrm{a}}=\mu_{\mathrm{A}}+q_{\mathrm{a}} \Phi_{\mathrm{a}, \mathrm{eq}}^{\exp }+\beta^{-1} \ln \left(c_{\mathrm{a}}\right),
$$


where $c_{\mathrm{a}}$ is the molar concentration of the ions in solution and $\beta^{-1}=k_{\mathrm{B}} T$.

As noted in the introduction, whenever the interfacial capacitance is independent of the applied potential - as e.g. in many implicit solvent models for high electrolyte concentrations [13, 16, 68] (see also section 1 in the Supporting Information (SI)) $-G_{\text {surf }}^{\alpha}$ in eq. (1) can be described with a second order polynomial in the number of electrons. In this case, the grand canonical, charge-equilibrated excess energy (constant potential conditions) decomposes into an excess energy term valid at the PZC - which is linear in the applied potential $\Phi_{E}$ and identical to the CHE expressions [16, 19,66] - and an additional, generic DL energy contribution due to capacitive charging [13, 16, 63]. Furthermore, mean-field sampling of configurations $\alpha$ at fixed adsorbate coverages $\theta_{\mathrm{a}}=N_{\mathrm{a}}^{\alpha} / N_{\text {sites }}$ can provide an approximate interface free energy landscape as a function of the coverage and the potential, from which any thermodynamic quantity can be inferred, in principle [13].

In the following, we will use expressions that are normalized to the number of surface sites (denoted by lower case letters), such that the mean-field CHE excess energy (per surface site) reads

$$
g_{\mathrm{exc}, 0}^{\theta_{\mathrm{a}} \mathrm{CHE}}=g_{\mathrm{exc}, 0}^{\text {clean }}+\theta_{\mathrm{a}} \bar{G}_{\mathrm{ads}, 0}^{\theta_{\mathrm{a}}}-\theta_{\mathrm{a}} \beta^{-1} \ln \left(c_{\mathrm{a}}\right)+\theta_{\mathrm{a}} q_{\mathrm{a}}\left(\Phi_{E}-\Phi_{\mathrm{a}, \mathrm{eq}}^{\mathrm{exp}}\right),
$$

where $g_{\text {exc }, 0}^{\text {clean }}$ is the excess energy of the clean surface and $\bar{G}_{\text {ads }, 0}^{\theta_{a}}$ the average adsorption energy per adsorbate, at coverage $\theta_{\mathrm{a}}$. The subscript 0 denotes values determined at the PZC. Both terms can be approximated e.g. from (charge-neutral) DFT calculations via

$$
\begin{aligned}
& g_{\text {exc }, 0}^{\text {clean }} \approx \frac{1}{N_{\text {sites }}}\left[E_{\text {surf }, 0}^{\text {cleat }, 0}-N_{\mathrm{s}} E_{\mathrm{s}, \text { bulk }}^{\mathrm{DFT}}\right] \\
& \bar{G}_{\mathrm{ads}, 0}^{\theta_{\mathrm{a}}} \approx\left\langle\frac{1}{N_{\mathrm{a}}^{\alpha}}\left[E_{\text {surf }, 0}^{\alpha, \mathrm{DT}}+\Delta F_{\text {surf }, \text { vib }}^{\alpha, \text { cor }}-E_{\text {surf }, 0}^{\text {clean, DFT }}-N_{\mathrm{a}}^{\alpha} \mu_{\mathrm{A}}^{\mathrm{DFT}}\right]\right\rangle_{\mathrm{MFT}} .
\end{aligned}
$$

$E_{\text {surf }, 0}^{\alpha, \mathrm{DFT}}$ and $E_{\text {surf, } 0}^{\text {cleaf }}$ are the DFT $0 \mathrm{~K}$ energies of the adsorbate-covered system consisting of $N_{\mathrm{a}}^{\alpha}$ adsorbates and $N_{\mathrm{s}}$ substrate atoms and of the pristine substrate system $\left(N_{\mathrm{s}}\right.$ substrate atoms). $E_{\mathrm{s}, \text { bulk }}^{\mathrm{DFT}}$ is the DFT $0 \mathrm{~K}$ bulk energy per atom of the substrate material. Here and in the following we assume all DFT surface energetics to be obtained using implicit solvation models. The corresponding terms could also be evaluated with explicit interfacial water to capture specific solvation effects. This can evidently affect the numerical values of both quantities, but would not affect the general thermodynamic description within the present work. The MF average $\langle\cdot\rangle_{\mathrm{MFT}}$ at fixed coverage $\theta_{\mathrm{a}}=N_{\mathrm{a}}^{\alpha} / N_{\text {sites }}$ can be efficiently approximated by random sampling or via the use of special quasi random structures [69-71]; for the considered showcase of halides on $\operatorname{Ag}(111)$ using only maximally isotropic structures at different coverages yielded already a good agreement between predicted and experimental CV peak shapes [13]. As none of the present discussion depends on the way these MF averages are determined (they might as well be determined by a fit to experimental data) we will refer to these averages at given coverage $\theta_{\mathrm{a}}$ simply by a corresponding superscript. In the case of a first-principles parametrization (e.g. based on eqs. (5), (6) ) inaccuracies due to the use of 0 $\mathrm{K}$ energy differences are largely corrected by addition of the term $\Delta F_{\text {surf,vib }}^{\alpha, \text { corr }}$ - the vibrational free energy contributions of the adsorbates only. As noted previously, within the CHE+DL approximation [13, 16, 63] the DL energy contributions per site due to capacitive charging are 
simply given by a plate-capacitor-like term

$$
\underline{g_{\mathrm{exc}}^{\theta_{\mathrm{a}} \mathrm{DL}}}=\underline{-\frac{1}{2} A_{\text {site }} C_{0}^{\theta_{\mathrm{a}}}\left(\Phi_{E}-\Phi_{0}^{\theta_{\mathrm{a}}}\right)^{2}},
$$

with the area per site $A_{\text {site }}=A / N_{\text {sites }} . \Phi_{0}^{\theta_{\mathrm{a}}}$ is the PZC (aka work function of the interface in solution) and $C_{0}^{\theta_{\mathrm{a}}}$ the area-normalized DL capacitance at the PZC for the coverages $\theta_{\mathrm{a}}$. Both intensive quantities can be determined again from ab initio calculations, e.g. with an implicit solvent model. In the context of the discussion here, they are to be seen as system-specific parameters which can vary for different adsorbates and experimental conditions. Finally, the MF free energy landscape per site as a function of $\theta_{\mathrm{a}}$ and $\Phi_{E}$ is given by

$$
g_{\mathrm{exc}}^{\theta_{\mathrm{a}}, \mathrm{MFT}} \approx g_{\mathrm{exc}, 0}^{\theta_{\mathrm{a}}, \mathrm{CHE}}+\underline{g_{\mathrm{exc}}^{\theta_{a}, \mathrm{DL}}}-T s_{\mathrm{conf}}^{\theta_{\mathrm{a}}},
$$

with $s_{\text {conf }}^{\theta_{a}}$ corresponding to the MF configurational entropy term

$$
s_{\text {conf }}^{\theta_{\mathrm{a}}}=-\theta_{\mathrm{a}}^{\max } k_{\mathrm{B}}\left[\left(\frac{\theta_{\mathrm{a}}}{\theta_{\mathrm{a}}^{\max }}\right) \ln \left(\frac{\theta_{\mathrm{a}}}{\theta_{\mathrm{a}}^{\max }}\right)+\left(1-\frac{\theta_{\mathrm{a}}}{\theta_{\mathrm{a}}^{\max }}\right) \ln \left(1-\frac{\theta_{\mathrm{a}}}{\theta_{\mathrm{a}}^{\max }}\right)\right],
$$

where $\theta_{\mathrm{a}}^{\max }$ is the maximum achievable coverage (per site) for the adsorbates a [13].

Above, and in the following, all DL-charging-related terms are underlined to make the impact of the latter more clear.

\subsection{Equilibrium interface compositions and thermodynamic CVs}

The equilibrium surface coverage $\bar{\theta}_{\mathrm{a}}\left(\Phi_{E}\right)$ at given applied electrode potential $\Phi_{E}$ minimizes $g_{\mathrm{exc}}^{\theta_{\mathrm{a}} \mathrm{MFT}}\left(\Phi_{E}\right)$ (eq. (8) with respect to $\theta_{\mathrm{a}}$. Knowledge of $\bar{\theta}_{\mathrm{a}}\left(\Phi_{E}\right)$ then directly yields the expected number of electrons $n_{e}^{\text {abs }}$ per site as a function of the potential via

$$
n_{e}^{\text {abs, }, \bar{\theta}_{\mathrm{a}}}=\bar{\theta}_{\mathrm{a}} \frac{q_{\mathrm{a}}}{e}-\frac{1}{e} A_{\mathrm{site}} C_{0}^{\bar{\theta}_{\mathrm{a}}}\left(\Phi_{E}-\Phi_{0}^{\bar{\theta}_{\mathrm{a}}}\right),
$$

from which thermodynamic CV currents $j$ can be derived [13]. At a fixed scan rate $v=\frac{\mathrm{d}}{\mathrm{d} t} \Phi_{E}$, this current can be written as $j=v C_{\text {pseudo, }}$, where the pseudocapacitance $C_{\text {pseudo }}$ is scan-rate-independent. Within the MF model, $C_{\mathrm{pseudo}}^{\mathrm{MFT}}=-e \frac{\mathrm{d}}{\mathrm{d} \Phi_{E}} n_{e}^{\mathrm{abs}, \bar{\theta}_{\mathrm{a}}}$ and thus given by

$$
\begin{aligned}
C_{\mathrm{pseudo}}^{\mathrm{MFT}} & =\underline{A_{\text {site }} C_{0}^{\bar{\theta}_{\mathrm{a}}}}+C_{\mathrm{pseudo}}^{\mathrm{MFT}, \text { orp }} \\
& =\underline{A_{\text {site }} C_{0}^{\bar{\theta}_{\mathrm{a}}}}-e l_{\mathrm{a}}^{\mathrm{MFT} \frac{\mathrm{d}}{\mathrm{d} \Phi_{E}} \bar{\theta}_{\mathrm{a}} .}
\end{aligned}
$$

$l_{\mathrm{a}}^{\mathrm{MFT}}$ is the electrosorption valency [13-16] - the number of exchanged electrons per adsorbate - which is given by

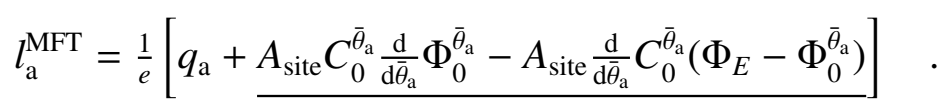

Note that expression 11 includes a double-layer charging, baseline contribution $A_{\text {site }} C_{0}^{\bar{\theta}_{\mathrm{a}}}$ plus a contribution due to electrosorption which we refer to as $C_{\mathrm{pseudo}}^{\mathrm{MFT} \text { sorp }}$. The latter is naturally proportional to the change in surface coverage $\left(\frac{\mathrm{d}}{\mathrm{d} \Phi_{E}} \bar{\theta}_{\mathrm{a}}\right)$ times the electrosorption valency $l_{\mathrm{a}}^{\mathrm{MFT}}$. 


\section{Results}

Until now we have put much attention to clarifying how the respective mean-field equations derive from an $a b$ initio thermodynamics-based ansatz. However, as this mean-field description is completely generic, these equations and the parameters therein form a general theory of thermodynamic CVs, where all mean-field parameters are to be seen as fundamental system-inherent descriptors. All CHE terms are thereby terms associated with the energetics at the PZC, and all underlined DL terms arise from the capacitive charging of the interface, aka the second order response to the application of a potential while leaving surface coverages fixed.

\subsection{Peaks}

$\bar{\theta}_{\mathrm{a}}$ minimizes ${ }_{\mathrm{g}} \overline{\mathrm{e}}_{\mathrm{axc}} \mathrm{MFT}$ and is thus given by

$$
\frac{\mathrm{d}}{\mathrm{d} \bar{\theta}_{\mathrm{a}}} g_{\mathrm{exc}}^{\bar{\theta}_{\mathrm{a}} \mathrm{MFT}}=0=\epsilon^{\bar{\theta}_{\mathrm{a}}}+\beta^{-1} \ln \left(\frac{\bar{\theta}_{\mathrm{a}}}{\bar{\theta}_{\mathrm{a}}^{\max }} /\left(1-\frac{\bar{\theta}_{\mathrm{a}}}{\bar{\theta}_{\mathrm{a}}^{\max }}\right)\right),
$$

which can be rewritten into the typical form of an adsorption isotherm as

$$
\bar{\theta}_{\mathrm{a}}=\theta_{\mathrm{a}}^{\max }\left[1+\exp \left(\beta \epsilon^{\bar{\theta}_{\mathrm{a}}}\right)\right]^{-1} .
$$

While eq. 14 defines $\bar{\theta}_{\mathrm{a}}\left(\Phi_{E}\right)$ in general only implicitly, it is possible to derive an analytic expression for the inverse function $\Phi_{E}^{\bar{\theta}_{a}}:=\Phi_{E}\left(\bar{\theta}_{\mathrm{a}}\right)$, which is given as the solution to the quadratic equation (in $\Phi_{E}$ ) defined by eq. (13) and with

$$
\begin{aligned}
& \epsilon^{\bar{\theta}_{\mathrm{a}}}=\frac{\mathrm{d}}{\mathrm{d} \bar{\theta}_{\mathrm{a}}}\left(g_{\mathrm{exc}, 0}^{\bar{\theta}_{\mathrm{a}}, \mathrm{CHE}}+\underline{g_{\mathrm{exc}}^{\bar{\theta}_{2}, \mathrm{DL}}}\right) \\
& =a\left(\Phi_{E}-\Phi_{0}^{\bar{\theta}_{a}}\right)^{2}+b\left(\Phi_{E}-\Phi_{0}^{\bar{\theta}_{a}}\right)+\mathcal{E} \\
& a=\underline{\left[-\frac{1}{2} A_{\text {site }} \frac{\mathrm{d}}{\mathrm{d} \bar{\theta}_{\mathrm{a}}} C_{0}^{\bar{\theta}_{\mathrm{a}}}\right]} \\
& b=q_{\mathrm{a}}+\underline{\left[A_{\text {site }} C_{0}^{\bar{\theta}_{\mathrm{a}}} \frac{\mathrm{d}}{\mathrm{d} \bar{\theta}_{\mathrm{a}}} \Phi_{0}^{\bar{\theta}_{\mathrm{a}}}\right]}=q_{\mathrm{a}}\left(1+\underline{\delta}_{\mathrm{a}}\right) \\
& \mathcal{E}=\bar{G}_{\mathrm{ads}, 0}^{\bar{\theta}_{\mathrm{a}}}+\bar{\theta}_{\mathrm{a}} \frac{\mathrm{d}}{\mathrm{d} \bar{\theta}_{\mathrm{a}}} \bar{G}_{\mathrm{ads}, 0}^{\bar{\theta}_{\mathrm{a}}}-\beta^{-1} \ln \left(c_{\mathrm{a}}\right)+q_{\mathrm{a}}\left(\Phi_{0}^{\bar{\theta}_{\mathrm{a}}}-\Phi_{\mathrm{a}, \mathrm{eq}}^{\mathrm{exp}}\right),
\end{aligned}
$$

The general solution to this second order equation in $\Phi_{E}$ (eq. (13), (15)) however hampers meaningful further analysis and we restrict ourselves in the following to the case where $a=0$ in eq. 15 . This approximation implicitly assumes $\frac{\mathrm{d}}{\mathrm{d} \bar{\theta}_{\mathrm{a}}} C_{0}^{\bar{\theta}_{\mathrm{a}}}=0$ and thus refers to systems, where the interfacial capacitance is independent on the surface coverage $\left(C_{0}^{\bar{\theta}_{\mathrm{a}}}=C_{0}\right)$ or where this is a good approximation. In this case

$$
\begin{aligned}
& \Phi_{E}^{\bar{\theta}_{\mathrm{a}}}=-\frac{1}{q_{\mathrm{a}}}\left[\mathcal{G}+q_{\mathrm{a}} \Delta_{\mathrm{a}}\right]=\Phi_{E}^{\bar{\theta}_{\mathrm{a}} \mathrm{CHE}}-\underline{\Delta}_{\mathrm{a}} \\
& \mathcal{G}=\mathcal{E}-q_{\mathrm{a}} \Phi_{0}^{\bar{\theta}_{\mathrm{a}}}+\beta^{-1} \ln \left(\frac{\bar{\theta}_{\mathrm{a}}}{\theta_{\mathrm{a}}^{\mathrm{max}}} /\left(1-\frac{\bar{\theta}_{\mathrm{a}}}{\bar{\theta}_{\mathrm{ax}}^{\max }}\right)\right) \\
& \underline{a}_{\mathrm{a}}=\underline{\delta}_{\mathrm{a}} \Delta \Phi_{E} \text { with } \Delta \Phi_{E}=\Phi_{E}^{\bar{\theta}_{\mathrm{a}}}-\Phi_{0}^{\bar{\theta}_{\mathrm{a}}},
\end{aligned}
$$

where $\underline{\delta}_{a}$ is a unitless measure for DL effects as defined in eq. (17). Note, that the CHE result is given by the first term in eq. 190 with $\Phi_{E}^{\bar{\theta}_{a}, C H E}=-\frac{\mathcal{G}}{q_{a}}$, where $\mathcal{G}$ is the differential, adsorptionenergy-like per adsorbate quantity defined in eq. (20). Inclusion of the (underlined) DL terms 
shifts $\Phi_{E}^{\bar{\theta}_{\mathrm{a}}, \mathrm{CHE}}$ thus by an amount of $-\underline{\Delta}_{\mathrm{a}}=-\underline{\delta}_{\mathrm{a}} \Delta \Phi_{E}$ at given coverages $\bar{\theta}_{\mathrm{a}}$ with $\Delta \Phi_{E}=\Phi_{E}^{\bar{\theta}_{\mathrm{a}}}-\Phi_{0}^{\bar{\theta}_{\mathrm{a}}}$ the difference between the applied potential and the PZC $\Phi_{0}^{\bar{\theta}_{\mathrm{a}}}$. Note that $\underline{\delta}_{\mathrm{a}}=D_{\mathrm{a}} C_{0}$ is the product of an adsorbate-dipole-related quantity $D_{\mathrm{a}}=\frac{A_{\text {site }}}{q_{\mathrm{a}}} \frac{\mathrm{d}}{\mathrm{d} \bar{\theta}_{\mathrm{a}}} \Phi_{0}^{\bar{\theta}_{\mathrm{a}}}$ and the DL capacitance $C_{0}$ (assumed independent on coverage already before). Thus the DL-induced shifts in the electrosorption potential can be rationalized by a dipole field interaction with the field in the double layer $\propto C_{0} \Delta \Phi_{E}$. This term corresponds to the interface charge at applied potential that is used as relevant variable in other works [18]. Furthermore, $q_{\mathrm{a}} \delta_{\mathrm{a}}$ relates directly to the electrosorption valency via $e l_{\mathrm{a}}^{\mathrm{MFT}}=q_{\mathrm{a}}\left(1+\underline{\delta}_{\mathrm{a}}\right.$ ) (cf. eq. 12 for $\frac{\mathrm{d}}{\mathrm{d} \bar{\theta}_{\mathrm{a}}} C_{0}^{\bar{\theta}_{\mathrm{a}}}=0$ and eq. (17)). The close connection between electrosorption valency and dipole has been clarified previously [15, 16]. Although adsorbate dipoles and interfacial fields are more common descriptors when discussing adsorbates at electrochemical interfaces, we use here mostly the unitless quantity $\underline{\delta}_{\mathrm{a}}$, as it represents a direct measure of the size of dipole-field interactions (at given DL capacitance) relative to the work by the electron transfer process upon adsorption, and it relates in a straightforward way to $l_{\mathrm{a}}^{\mathrm{MFT}}$.

Evidently, $\Phi_{E}^{\bar{\theta}_{\mathrm{a}}}$ (eq. 19 ) can be approximated as a perturbation to the $\mathrm{CHE}$ result $\Phi_{E}^{\bar{\theta}_{\mathrm{a}}, \mathrm{CHE}}$ via

$$
\Phi_{E}^{\bar{\theta}_{\mathrm{a}}} \approx \Phi_{E}^{\bar{\theta}_{\mathrm{a}}, \mathrm{CHE}}-\underline{\delta}_{\mathrm{a}} \Delta \Phi_{E}^{\mathrm{CHE}},
$$

where $\Delta \Phi_{E}^{\mathrm{CHE}}=\Phi_{E}^{\bar{\theta}_{\mathrm{a}}, \mathrm{CHE}}-\Phi_{0}^{\bar{\theta}_{\mathrm{a}}}$ is the potential drop in the double layer, as estimated from the CHE electrosorption potentials $\Phi_{E}^{\bar{\theta}_{a}, \mathrm{CHE}}$. With $\mathrm{Cl} @ \mathrm{Ag}(111)$ having electrosorption valencies of $\sim-0.5$ [16] we can directly derive $\left|\underline{\delta}_{\mathrm{a}}\right| \approx 50 \%$ and thus estimate the DL-charging-related shift of the CHE peak position as $\approx 0.5\left|\Delta \Phi_{E}^{\mathrm{CHE}}\right|$, which is easily in the range of hundreds of $\mathrm{meV}$, and thus far from negligible团

3.1.1. Peak position As discussed above, CVs are typically dominated by the electrosorption peak $C_{\text {pseudo }}^{\mathrm{MFT} \text {,sorp }}$ (eq. 11 ). Whenever $l_{\mathrm{a}}^{\mathrm{MFT}}$ depends only weakly on the coverage, the position and width of the $\mathrm{CV}$ peak are hardly affected by its neglect, as it enters only as a multiplicative constant in eq. 111 . Thus, we focus in the following on the quantity $C=\sigma \frac{\mathrm{d}}{\mathrm{d} \Phi_{E}} \bar{\theta}_{\mathrm{a}}$, with $\sigma=\frac{-q_{\mathrm{a}}}{\left|q_{\mathrm{a}}\right|}$. This expression yields positive electrosorption peaks, independent on the sign of the adsorbate charge $q_{\mathrm{a}}$ for physically sound, i.e. convex free energy landscapes $\|$.

Thus, CV peak position and shape can be evaluated from the moments of $C$ via the integrals

$$
\left\langle\Phi_{E}^{n}\right\rangle=\frac{1}{N} \int_{-\infty}^{\infty} \mathcal{C} \cdot \Phi_{E}^{n} \mathrm{~d} \Phi_{E}
$$

and the normalization $N=\int_{-\infty}^{\infty} C \cdot \mathrm{d} \Phi_{E}$. These can be rewritten as integrals over the coverage

$\ddagger$ As the peak position is directly related to adsorption energies as e.g. used in kinetic theories, this error has a dramatic impact on the predictive quality of those.

$\S$ The appropriate inclusion of the electrosorption valency is performed in the SI in section 3 . The resulting equations do however not allow further insights as gained by the presented analysis.

\| Systems where adsorption energies decrease with increasing coverage can lead to non-convex free energy landscapes in mean-field theory, which we do not consider here. 
$\bar{\theta}_{\mathrm{a}}$ (see section 2 in the SI for more details), as

$$
\left\langle\Phi_{E}^{n}\right\rangle=\frac{1}{\theta_{\mathrm{a}}^{\max }} \int_{0}^{\theta_{\mathrm{a}}^{\max }}\left(\Phi_{E}^{\bar{\theta}_{a}}\right)^{n} \mathrm{~d} \bar{\theta}_{\mathrm{a}} .
$$

The peak position $P$ on an absolute potential scale is given by the first moment

$$
P=\left\langle\Phi_{E}^{\bar{\theta}_{a}}\right\rangle \approx\left\langle\Phi_{E}^{\bar{\theta}_{a}, \mathrm{CHE}}\right\rangle-\left\langle\underline{\Delta}_{\mathrm{a}}\right\rangle,
$$

which can be readily computed from the coverage-dependent adsorption energetics (cf. eqs. (19), (24)). This result clarifies the above mentioned influence of the dipole-field interaction $\underline{\Delta}_{\mathrm{a}}$ on the expected peak position relative to the CHE estimate. Furthermore, it is straightforward to derive peak shifts induced by a variation of the concentration $c_{\mathrm{a}}$ via the identities

$$
\begin{aligned}
\frac{\mathrm{d}}{\operatorname{d} \log \left(c_{\mathrm{a}}\right)}\left\langle\Phi_{E}^{n}\right\rangle & =\left\langle n \Phi_{E}^{n-1} \frac{\partial \Phi_{E}^{\bar{\theta}_{\mathrm{a}}}}{\partial \log \left(c_{\mathrm{a}}\right)}\right\rangle, \\
\frac{\partial \Phi_{E}^{\bar{a}_{\mathrm{a}}}}{\partial \log \left(c_{\mathrm{a}}\right)} & \approx \frac{\beta^{-1} \ln (10)}{q_{\mathrm{a}}}\left(1-\underline{\delta}_{\mathrm{a}}\right) .
\end{aligned}
$$

The latter approximation is valid for small $\underline{\delta}_{\mathrm{a}}$ (cf. eq. (22) and section 2 in the SI). Evidently, the first term in eq. 27) describes the perfect Nernstian behaviour of the CHE energetics, whereas the DL terms induce Non-Nernstian shifts according to

$$
\frac{\mathrm{d}}{\mathrm{d} \log \left(c_{\mathrm{a}}\right)} P=\frac{\mathrm{d}}{\mathrm{d} \log \left(c_{\mathrm{a}}\right)}\left\langle\Phi_{E}^{\bar{\theta}_{\mathrm{a}}}\right\rangle \approx \frac{\beta^{-1} \ln (10)}{q_{\mathrm{a}}}\left(1-\left\langle\underline{\delta}_{\mathrm{a}}\right\rangle\right),
$$

which can be directly evaluated from the coverage dependence of the PZC and knowledge of the DL capacitance $C_{0}$ (cf. eq. (17)). Therefore, non-Nernstian peak shifts are directly related to non-ideal electrosorption valencies $e l_{\mathrm{a}}^{\mathrm{MFT}}=q_{\mathrm{a}}+q_{\mathrm{a}} \underline{\mathrm{S}}_{\mathrm{a}} \neq q_{\mathrm{a}}$, which depend on the adsorbate dipole and the interfacial capacitance, not however on the magnitude or direction of the field in the double layer (within the present approximations).

3.1.2. Proxies for peak position and peak shape In analogy to the previous analysis, peak widths can be analyzed via the second central moment, as done in the SI (sections 2,3 ), however, the intricate averages over the coverage hamper further insightful, analytical analysis. Therefore, we follow a simpler route to derive proxies for the position and peak shape of CVs by estimating above averages over the coverage $\langle\cdot\rangle$ by simple evaluation of the coverage-dependent terms at an intermediate coverage, e.g. the half maximum coverage. This procedure allows to derive in full rigour general and more intelligible proxies for $\mathrm{CV}$ peak properties, as elaborated in the SI. We refer the interested reader to the respective section 4 in the SI, also to understand better the conditions under which the various approximations made are expected to remain valid. All derived expressions are reported in a self-contained way for $\mathrm{CV}$ peak position and shape (table 1) as well as for their variation when changing the ion concentration $c_{\mathrm{a}}$ and the double layer capacitance $C_{0}$ (table 2).

Indeed, the respective equations show that changing the ion concentration $c_{\mathrm{a}}$ can affect $\mathrm{CV}$ peak positions and shapes, and these changes can be understood from the changes in dipole-field-like interactions which rationalize the respective descriptors (see more detailed discussion later on). 
Table 1. Approximate expressions for the CV peak position $P$, the potential drop in the DL $\Delta \Phi_{E}$ and the CV peak shape as defined by its width $W$ and height $H$. While all other variables are as introduced in the main text, the variables $\sigma=-\frac{q_{\mathrm{a}}}{\left|q_{\mathrm{a}}\right|}= \pm 1$ and $K>0=O(1)$ can be understood from the detailed derivations in section 4 of the SI. For halides on $\operatorname{Ag}(111)$ $K \approx 0.27$. All coverage-dependent terms are to be evaluated at half maximum coverage $\left(\bar{\theta}_{\mathrm{a}}=\frac{1}{2} \theta_{\mathrm{a}}^{\max }\right)$.

\begin{tabular}{|c|c|c|}
\hline property & proxy & \\
\hline $\begin{array}{l}\text { CHE peak } \\
\text { position }\end{array}$ & $\begin{aligned} P^{\mathrm{CHE}}= & -\frac{\mathcal{G}}{q_{\mathrm{a}}} \\
\mathcal{G}= & \bar{G}_{\mathrm{ads}, 0}+\bar{\theta}_{\mathrm{a}} \frac{\mathrm{d}}{\mathrm{d} \bar{\theta}_{\mathrm{a}}} \bar{G}_{\mathrm{ads}, 0} \overline{\bar{\theta}}_{\mathrm{a}}-q_{\mathrm{a}} \Phi_{\mathrm{a}, \mathrm{eq}}^{\exp } \\
& -\beta^{-1} \ln \left(c_{\mathrm{a}}\right)+\beta^{-1} \ln \left(\frac{\bar{\theta}_{\mathrm{a}}}{\bar{\theta}_{\mathrm{a}}^{\max }} /\left(1-\frac{\bar{\theta}_{\mathrm{a}}}{\theta_{\mathrm{a}}^{\max }}\right)\right)\end{aligned}$ & (29) \\
\hline $\begin{array}{l}\text { CHE+DL } \\
\text { peak } \\
\text { position }\end{array}$ & $P=P^{\mathrm{CHE}}-\underline{\delta}_{\mathrm{a}} \Delta \Phi_{E}$ & (30) \\
\hline $\begin{array}{l}\text { DL poten- } \\
\text { tial drop }\end{array}$ & $\Delta \Phi_{E}=\Phi_{E}^{\bar{\theta}_{a}}-\Phi_{0}^{\bar{\theta}_{\mathrm{a}}}=P-\Phi_{0}^{\bar{\theta}_{\mathrm{a}}} \approx \Delta \Phi_{E}^{\mathrm{CHE}}=P^{\mathrm{CHE}}-\Phi_{0}^{\bar{\theta}_{a^{2}}}$ & $(31)$ \\
\hline $\begin{array}{l}\text { CHE+DL } \\
\text { width }\end{array}$ & $\begin{aligned} W & =W^{\mathrm{CHE}}+W^{\mathrm{DL}} \\
W^{\mathrm{CHE}} & =\sigma K\left(\frac{\mathrm{d}}{\mathrm{d} \bar{\theta}_{\mathrm{a}}} \Phi_{E}^{\bar{\theta}_{a}, \mathrm{CHE}}\right) \\
W^{\mathrm{DL}} & =-\sigma K \cdot \frac{\mathrm{d}}{\mathrm{d} \bar{\theta}_{a}} \Delta_{\mathrm{a}} \\
& =-W^{\mathrm{CHE}} \cdot \underline{\delta}_{\mathrm{a}}-\frac{\left|q_{\mathrm{a}}\right| K}{A_{\text {site }} C_{0}} \cdot \underline{\delta}_{\mathrm{a}}^{2}-\sigma K \frac{\mathrm{d}}{\mathrm{d} \bar{\theta}_{\mathrm{a}}} \underline{\delta}_{\mathrm{a}} \cdot \Delta \Phi_{E}\end{aligned}$ & $\begin{array}{l}(32) \\
(33) \\
(34)\end{array}$ \\
\hline $\begin{array}{l}\text { CHE+DL } \\
\text { height }\end{array}$ & $H=\left|q_{\mathrm{a}}\left(1+\underline{\delta}_{\mathrm{a}}\right)\right| \frac{K}{W}$ & $(35)$ \\
\hline
\end{tabular}

In the same way, a change of the DL capacitance $C_{0}$ can introduce changes in peak position and shape. As $C_{0}$ changes with electrolyte concentrations but also with electrolyte ion type [17] due to variations in ionic solvation shell sizes [18], the derived dependencies on $C_{0}$ are highly important to rationalize non-trivial changes under different experimental conditions.

We will provide a more detailed discussion and evaluation of these proxies in table 1 and 2 later on. 
Table 2. Proxies for the concentration dependence $\frac{\mathrm{d}}{\mathrm{d} \log \left(c_{\mathrm{a}}\right)}$ and the capacitance dependence $\frac{\mathrm{d}}{\mathrm{d} C_{0}}$ for the $\mathrm{CV}$ peak position $P$, the width $W$ and the height $H$.

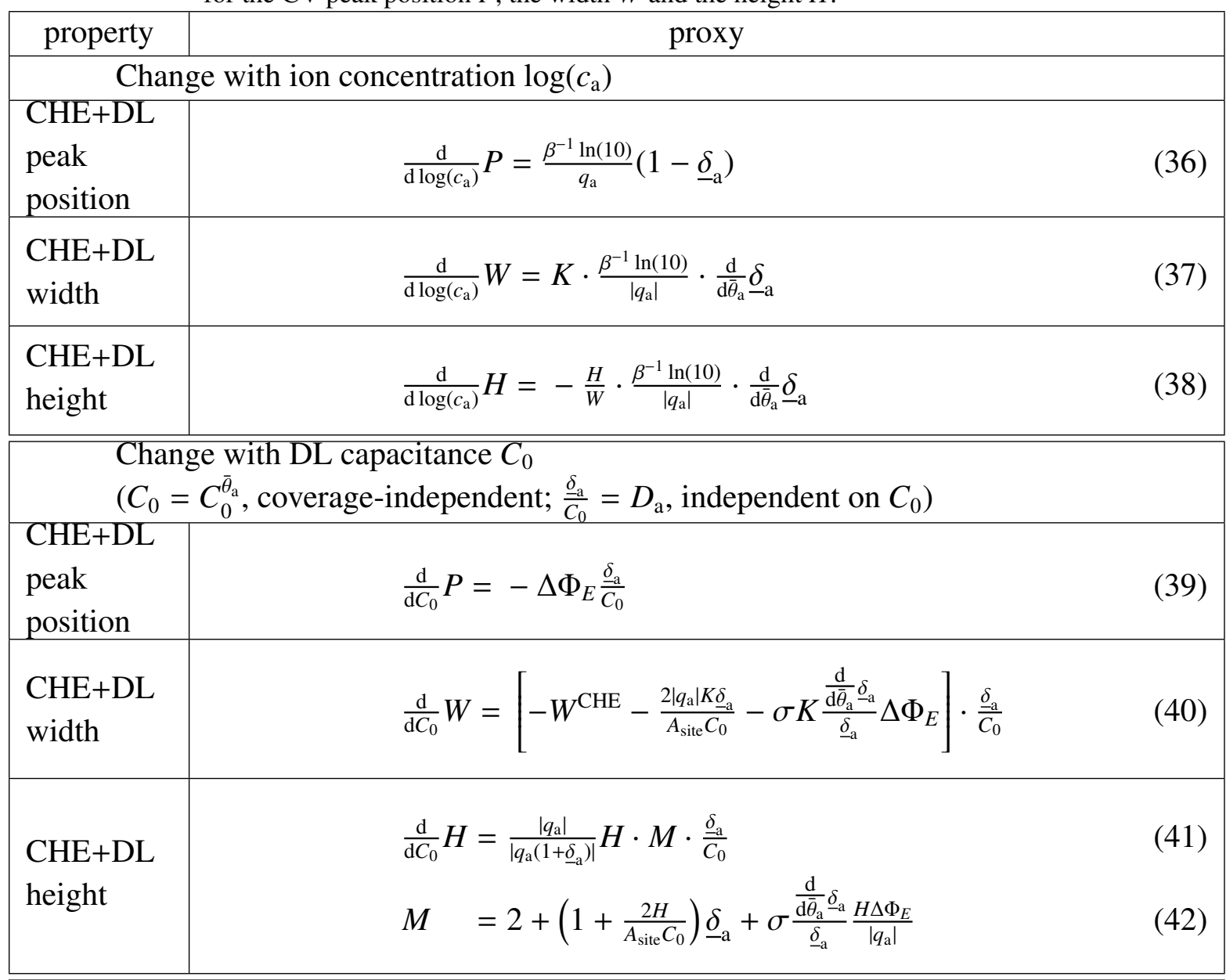




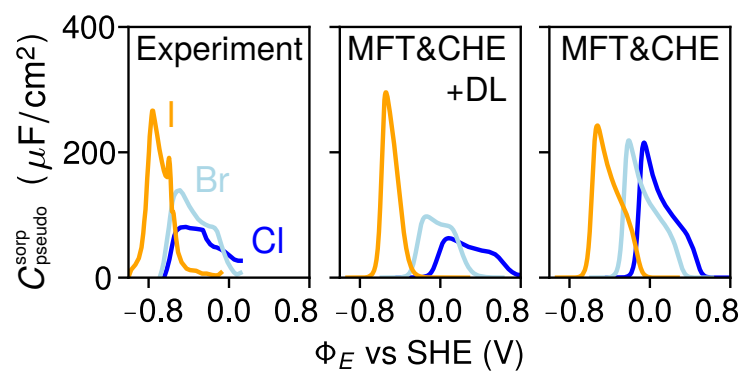

Figure 1. Experimental [72] and theoretical [13] CV peaks for $\operatorname{Ag}(111)$ in halide containing solutions at ion concentrations of $c_{\mathrm{a}}=0.5 \mathrm{mM}$. The theoretical results are based on the present mean-field description at the CHE+DL and CHE level of theory as parametrized in Ref. [13] based on DFT calculations in an implicit solvent model. All curves are without capacitive baseline currents and plot the scan-rate-independent property $C_{\text {pseudo }}^{\text {sorp }}$ (area-normalized values, see section 6 in the SI for more details). Only the theoretical CHE+DL results can replicate the experimental variations in peak shapes for $\mathrm{Cl}, \mathrm{Br}$, and $\mathrm{I}$, both qualitatively and quantitatively, which supports the importance of capacitive DL charging for these systems.

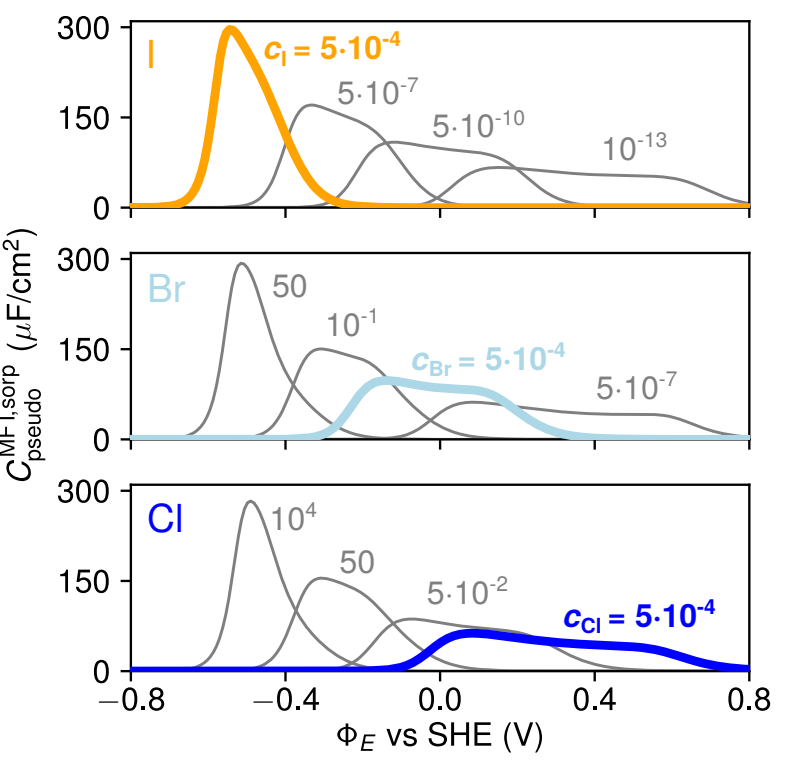

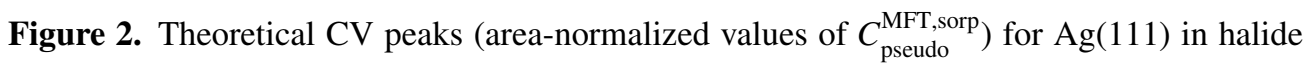
containing solutions as a function of the concentrations $c_{\mathrm{a}}$ (MFT\&CHE+DL parametrization from Ref. [13]). The CVs corresponding to the central panel of Fig. 1 at concentrations $c_{\mathrm{a}}=5 \cdot 10^{-4} \mathrm{M}$ are colored and bold. All additional grey peaks are simulated at the indicated molar concentrations (values partly unphysical). The plot demonstrates firstly a strong concentration-dependence of all halide CVs and and secondly that all halide peaks are expected in essence identical, whenever their position $P$ on the potential axis is the same, in agreement with the importance of the potential drop in the double layer as the shapedetermining factor [13].

\subsection{Accuracy validation for the proxies: halide electrosorption on Ag(111)}

DL effects were found relevant for understanding thermodynamic CVs of $\mathrm{Ag}(111)$ in halide containing solutions [13], as evidenced by comparing experimental results of Foresti et al. [72] with theoretical predictions of the present mean-field description at the CHE and 

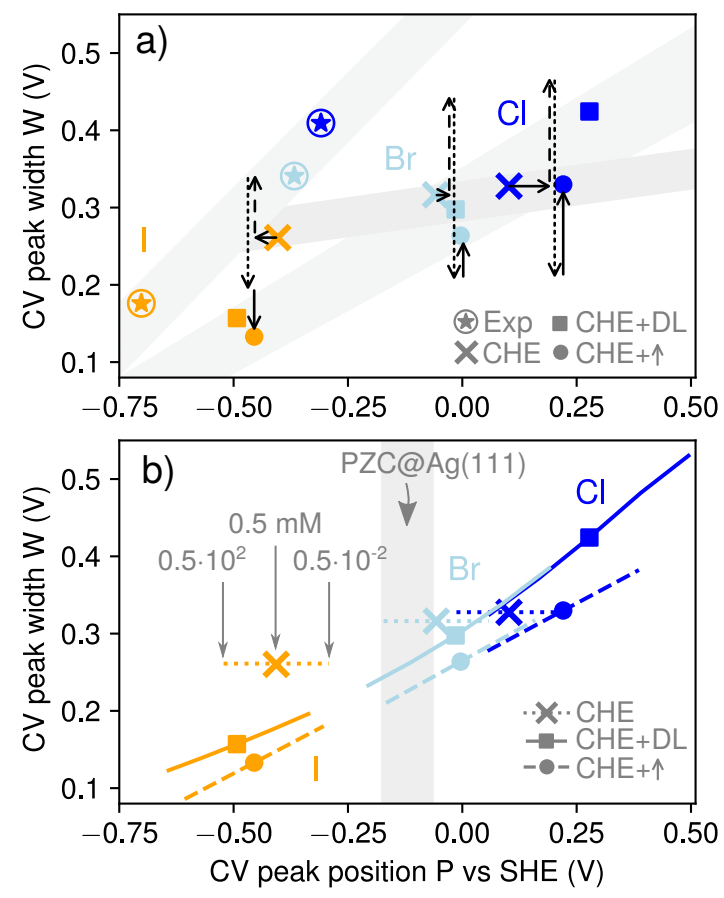

Figure 3. a) Experimental and theoretical peak positions $P$ and widths $W$ for $c_{\mathrm{a}}=0.5 \mathrm{mM}$, computed via first and second central moment of $C_{\text {pseudo }}^{\text {sorp }}\left(\Phi_{E}\right)$ (see text). CHE and CHE+DL results are plotted as crosses and squares. The DL-induced CHE perturbations are indicated as arrows (horizontal arrow: eq. 25); vertical dashed, dotted and solid arrows: first, second and third term in eq. (34)). Adding those to the CHE data points (CHE+ $\uparrow$, filled circles) explain approximately the CHE+DL results. The grey regions are a guide to the eye to highlight the underestimated correlation between positions $P$ and widths $W$ in the CHE model. b) $P$ and $W$ for the three theoretical methods in a concentration range of $c_{\mathrm{a}}=0.5 \cdot 10^{ \pm 2} \mathrm{mM}$. The grey region corresponds to the PZC of half-covered $\mathrm{Ag}(111)$ surfaces.

CHE+DL level of theory, parametrized from DFT calculations in an implicit solvent model (see Fig. 1 and Ref. [13]). The inability of the pure CHE description to replicate the experimental variations in the peak shapes, hints at the importance of capacitive DL charging for these systems.

This becomes even more clear, when studying the changes in the peak shapes with changing the ion concentrations $c_{\mathrm{a}}$ for the according CHE+DL mean field models, as reported in Fig. 23r. Results at the experimental conditions $\left(c_{\mathrm{a}}=5 \cdot 10^{-4} \mathrm{M}\right.$ [72], as in Fig. 11) are colored and bold, while results for selected alternative concentrations are in grey (molar concentrations as indicated, partly unphysical). The plot demonstrates the strong concentration-dependence of the according CVs and clarifies that all halide peak shapes are expected in essence identical, given that their position $P$ on the potential axis is the same. This can be easily understood from the previous discussions and the fact that all adsorbate-related descriptors but the adsorption energy are essentially the same for $\mathrm{Cl}, \mathrm{Br}$ and I (see Ref. [13] and Fig. SF1 in the SI).

In order to assess the accuracy of the derived proxies, we use these results for the $\mathrm{CV}$ II Details on the parameters in Ref. [13] and the SI, section 6 
peaks for $\mathrm{Ag}(111)$ in $\mathrm{Cl}, \mathrm{Br}$ and I solutions, to test in how far the differences between the $\mathrm{CHE}$ and the $\mathrm{CHE}+\mathrm{DL}$ results (Fig. 1) and the relevant changes with the concentration (Fig. 2) can be described and understood from the derived proxies. Unfortunately, the non-negligible degree of asymmetry in the peak shapes (see Figs. 1, 2p hampers an unambiguous definition of position $P$, width $W$ and height $H$. Therefore, we restrict ourselves to an analysis of $P$ and $W$, for which the above moments-based definition can provide unambiguous and well-defined results. Verification of the proxies for $P$ and $W$ will also validate the proxies for $H$ as it is the former two that enter the derivation of the peak-height-related proxies (see section 4 in the SI).

Figure 3 a reports peak positions $P$ and peak widths $W$ for the experiments (encircled stars), as well as for the ab initio parametrized mean-field models (CHE: crosses, CHE+DL: squares) with quadratic coverage dependencies (see Ref. [13] and section 6 in the SI), at an ion concentration of $c_{\mathrm{a}}=0.5 \mathrm{mM} . P$ and $W$ are computed from the mean and $2^{\text {nd }}$ central moment of the experimental and theoretical curves of $C_{\text {pseudo }}^{\text {sorp }}\left(\Phi_{E}\right)$ according to

$$
\begin{aligned}
P & =\frac{\int C_{\text {pseudo }}^{\text {sorp }}\left(\Phi_{E}\right) \cdot \Phi_{E} \mathrm{~d} \Phi_{E}}{\int C_{\text {pseudo }}^{\text {sorp }}\left(\Phi_{E}\right) \mathrm{d} \Phi_{E}} \\
W & =2 \sqrt{\frac{\int C_{\mathrm{pseudo}}^{\text {sorp }}\left(\Phi_{E}\right) \cdot\left(\Phi_{E}-P\right)^{2} \mathrm{~d} \Phi_{E}}{\int C_{\mathrm{pseudo}}^{\text {sorp }}\left(\Phi_{E}\right) \mathrm{d} \Phi_{E}}} .
\end{aligned}
$$

We then evaluate the accuracy of the proxies by adding to each $\mathrm{CHE}$ data point $\left(P^{\mathrm{CHE}}, W^{\mathrm{CHE}}\right)$ the approximate expressions for the DL-induced peak shift (eq. (25), horizontal vectors in Fig. 3 a) and the width change (eq. (34), vertical vectors). The individual contributions to the DL-induced width change (first, second and third term in eq. (34)) are illustrated as dashed, dotted and solid vertical arrows, respectively. All estimates are evaluated at half coverage $\left(0.5 \theta_{\mathrm{a}}^{\max }\right)$ and with $K=0.27$ in eq. (34) (see section 4 in the SI for the detailed derivation of this value). All approximate results that derive from adding to each CHE data point the respective, perturbative shifts are plotted as filled circles and denoted as

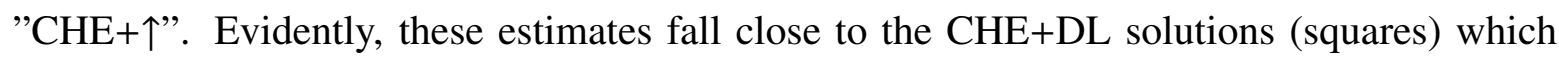
provides confidence in the accuracy of the derived proxies.

Shaded regions enclosing experimental and theoretical data points across the halide series support a strong overall dependence of peak widths $W$ on the peak position $P$ for the experiments, which is only fully captured by theory when including the DL energetics. Note, that the inclusion of only the first two terms of eq. (34) (dashed and dotted vertical arrows) leads to identical widths across the halide series. Thus only the latter term, which varies with the potential drop in the double layer $\left(\Delta \Phi_{E}\right)$, seems responsible for the variation of the halide peak shapes, in line with the results in Fig. 22 and the conclusions of Ref. [13].

Figure 3 b plots the theoretical values for $P$ and $W$ when changing the ion concentration by two orders of magnitude around the experimental value of $c_{\mathrm{a}}=0.5 \mathrm{mM}\left(c_{\mathrm{a}}=0.5\right.$. $10^{ \pm 2} \mathrm{mM}$ ). While the CHE method only yields trivial Nernstian shifts in the peak position, the $\mathrm{CHE}+\mathrm{DL}$ theory and their approximation $(\mathrm{CHE}+\uparrow)$ lead to non-trivial position and width changes (cf. Fig. 2). As analysed subsequently, the former is related to the fact that halides on $\mathrm{Ag}(111)$ exhibit a normal work function change (see Fig. SF1 in the SI). Furthermore, as the work function change per adsorbate increases with the coverage for these systems (see Fig. 
SF1 in the SI) eq. 37 results in a decreasing peak width with increasing $c_{\mathrm{a}}$ as $\frac{\mathrm{d}}{\mathrm{d} \overline{\mathrm{e}}_{\mathrm{a}}} \underline{\delta}_{\mathrm{a}}<0$, which explains the behaviour observed in Fig. $3 \mathrm{~b}$.

Figure $3 \mathrm{~b}$ includes as well the PZC of $\mathrm{Ag}(111)$ at half maximum coverage. At these potentials most DL-induced alterations to the $\mathrm{CHE}$ results are expected to vanish as there are nearly no interfacial fields present. Indeed, the concentration-dependencies of the $\mathrm{CHE}+\mathrm{DL}$ and $\mathrm{CHE}+\uparrow$ methods extrapolate nicely to the $\mathrm{CHE}$ results in this potential regime, which underlines again that the CHE results are intrinsically only valid at the PZC (cf. Figs. 1, 2).

Halides on $\operatorname{Ag}(111)$ exhibit significant coverage dependencies (e.g. for $C_{0}^{\bar{\theta}_{\mathrm{a}}}$ and $\underline{\delta}_{\mathrm{a}}$, see Fig. SF1 in the SI), however, their approximate treatment by evaluation at half maximum coverage and their complete neglect $\left(a=0 ; C_{0}^{\overline{\theta_{a}}}=C_{0}\right.$ in eq. 15 ) ) does not prevent the proxies from reproducing the $\mathrm{CHE}+\mathrm{DL}$ results even in a semi-quantitative way. This is even more so surprising, as $\left|\underline{\delta}_{\mathrm{a}}\right| \in 0.2 \ldots 0.5$ for halides on $\operatorname{Ag}(111)$ and thus not small compared to 1, which is assumed at certain steps of the derivations (see SI).

Therefore, we expect these proxies to remain valid also for other systems, where the approximations are typically less problematic than for the here discussed showcase system.

\subsection{Analysis of the proxies}

An insightful and accessible interpretation of the physical meaning and origin of the derived proxies in tables 1 and 2 can be achieved by analysing a Frumkin model, where the adsorption energy $\bar{G}_{\text {ads }, 0}^{\bar{\theta}_{\mathrm{a}}}$ is assumed to vary only linearly with the coverage, and where adsorbate dipoles are assumed as coverage-independent. Such systems show no shape dependence on $\log \left(c_{\mathrm{a}}\right)$, but exhibit Non-Nernstian peak shifts. The according analysis is not only interesting due to its straightforward interpretability, but also as it can be treated fully analytically. We refer the reader to section 5 in the SI for details.

3.3.1. The quantity $\underline{\delta}_{\mathrm{a}} \quad$ As already stated the quantity $\underline{\delta}_{\mathrm{a}}$ is a unitless measure of DL effects, and at the same time it relates trivially to the electrosorption valency via $e l_{\mathrm{a}}^{\mathrm{MFT}}=q_{\mathrm{a}}\left(1+\underline{\delta}_{\mathrm{a}}\right)$. Its meaning is thus very accessible and becomes even more clear when we reformulate e.g. the traditional chemical reaction equation for the electrosorption of $\mathrm{Cl}^{-}$

$$
\mathrm{Cl}^{-}+* \rightarrow \mathrm{Cl} *+e^{-}
$$

into a more general electrosorption equation, namely

$$
\mathrm{Cl}^{-}+* \rightarrow \mathrm{Cl}^{\underline{\delta_{\mathrm{Cl}}} *}+\left(1+\underline{\delta}_{\mathrm{Cl}}\right) e^{-}
$$

with $\underline{\delta}_{\mathrm{Cl}}$ being identical to the according quantity used throughout this work and with $\underline{\delta}_{\mathrm{Cl}} \approx-0.5$ [16]. Reaction equation (46) thus clarifies that some negative charge remains on the adsorbed $\mathrm{Cl}$ and thus only $\left(1+\underline{\delta}_{\mathrm{Cl}}\right) \approx 0.5$ electrons are actually exchanged upon the electrosorption process. Note, however, that this is only a simplified picture, as above chargeconserving reaction equations are problematic in view of a fully grand canonical picture where charge is not a conserved quantity. 
In general, $\underline{\delta}_{\mathrm{a}}$ discriminates between systems with normal and anomalous work function change: $\underline{\delta}_{\mathrm{a}}$ is negative for adsorbates with a normal work function change $\left(\frac{\mathrm{d}}{\mathrm{d} \bar{\theta}_{\mathrm{a}}} \Phi_{0}^{\bar{\theta}_{\mathrm{a}}}<0\right.$ for $q_{\mathrm{a}}>0$ and $\frac{\mathrm{d}}{\mathrm{d} \bar{\theta}_{\mathrm{a}}} \Phi_{0}^{\bar{\theta}_{\mathrm{a}}}>0$ for $\left.q_{\mathrm{a}}<0\right)$. On the other hand $\underline{\delta}_{\mathrm{a}}>0$ for adsorbates with anomalous work function change (cf. eq. (17)). Thus it makes a significant difference, whether adsorbates behave normally or anormally in this respect.

3.3.2. Peak positions $P$ Indeed the work function changes due to the adsorption of halides on metallic surfaces can be surprisingly complex [73-75], and it is thus certainly interesting to analyse what these results mean in terms of their electrosorption behaviour.

Based on the approach outlined in section 7 of the SI, we use published theoretical data [74] for $\bar{G}_{\text {ads }, 0}^{\bar{\theta}_{\mathrm{a}}}$ and $\frac{\mathrm{d}}{\mathrm{d} \bar{\theta}_{\mathrm{a}}} \Phi_{0}^{\bar{\theta}_{\mathrm{a}}}$ in combination with our own results for halides on $\operatorname{Ag}(111)$, in order to estimate the values of $\underline{\delta}_{\mathrm{a}}$ and $\Delta \Phi_{E}$ at interfacial capacitances $C_{0} \approx 40 \mu \mathrm{F} / \mathrm{cm}^{2}$ for Cl, $\mathrm{Br}$ and I and for a wide range of substrate materials (see section 7 in the SI for more details). This allows us directly to estimate their Non-Nernstian peak shifts as well as the accuracies of the CHE peak positions (eq. (30p), which depend only on the sign and order of magnitude of $\underline{\delta}_{\mathrm{a}}$ and the peak position relative to the PZC.

Figure 4 plots the derived numerical values for $\underline{\delta}_{\mathrm{a}}$ and $l_{\mathrm{a}}^{\mathrm{MFT}}=-\left(1+\underline{\delta}_{\mathrm{a}}\right)$ as a function of the vacuum work function of the substrate material. Evidently, for many adsorbate-substrate combinations, $\left|\underline{\delta}_{\mathrm{a}}\right|$ takes values that are significantly different from zero. In addition, negative and positive values are observed, in line with the fact that halides can induce normal and anomalous work function changes [73-75].

As a result of eqs. (28), (36), adsorbates with normal work function change exhibit larger peak shifts with $\log \left(c_{\mathrm{a}}\right)$ than expected from the ideal Nernst behaviour. The reverse is true for adsorbates with an anomalous work function shift. This is perfectly in line with the interpretations gained from the Frumkin model analysed in the SI, and the above intuitive chemical reaction equation (46): With $\underline{\delta}_{\mathrm{a}}$ being directly related to the electrosorption valency $l_{\mathrm{a}}^{\mathrm{MFT}}=\frac{q_{\mathrm{a}}}{e}\left(1+\underline{\delta}_{\mathrm{a}}\right)$, non-zero $\underline{\delta}_{\mathrm{a}}$ values are directly linked to a rescaled driving force for the adsorption process. Thus, systems with normal(anomalous) work function change exchange less(more) electrons upon adsorption, and thus necessitate larger(smaller) applied potentials to drive the adsorption process. While these results hold in general, e.g. independent on the charge of the adsorbate, we exemplify it in Fig. 4f for the negatively charged halides by having included also the shift with $\log \left(c_{\mathrm{a}}\right)$ on the right y-axis (eq. (36)), which evidently exhibits the described dependence and demonstrates the expected magnitude of Non-Nernstian behaviour for the considered systems (Nernstian: $\frac{\mathrm{d}}{\mathrm{d} \log \left(c_{\mathrm{a}}\right)} P=60 \mathrm{mV} /$ decade).

The combination of the $\underline{\delta}_{\mathrm{a}}$ values with the reported [74] adsorption energies $\bar{G}_{\text {ads }, 0}^{\bar{\theta}_{\mathrm{a}}}$ allows to assess directly the expected CHE errors in the peak positions $P-P^{\mathrm{CHE}}$ (eq. 30 ) as well as the peak shift with DL capacitance $\frac{\mathrm{d}}{\mathrm{d} C_{0}} P$ (eq. 39 ). Note that both proxies are trivially related via the DL capacitance $C_{0}$. The DL potential drop is approximated via $\Delta \Phi_{E} \approx P^{\mathrm{CHE}}-\Phi_{0}^{\bar{\theta}_{\mathrm{a}}}$ and with $P^{\mathrm{CHE}}$ from the theoretically reported values of $\bar{G}_{\text {ads }, 0}^{\bar{\theta}_{\mathrm{a}}}$ (eq. 29 ) and the PZC $\Phi_{0}^{\bar{\theta}_{\mathrm{a}}}$ from experimentally measured values, which are available for the major part of the studied substrates [76] (see SI, section 7). The results are plotted in Fig. 5f for an ion concentration in 


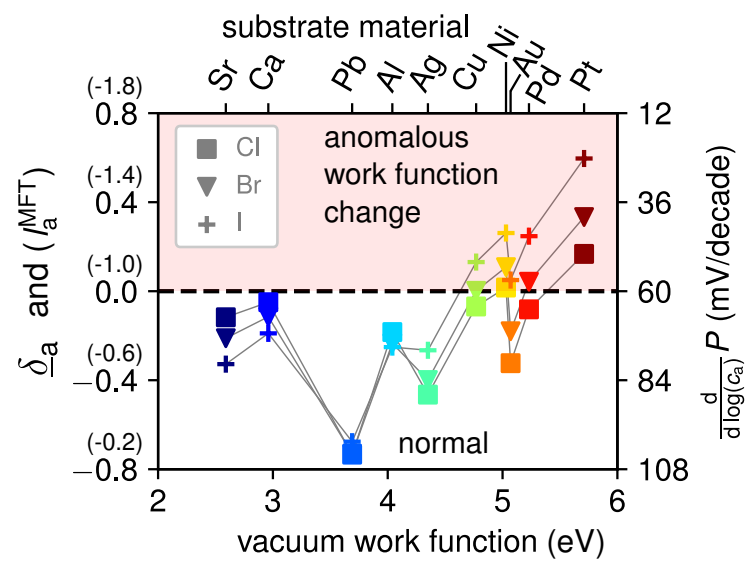

Figure 4. Numerical values for $\underline{\delta}_{\mathrm{a}}$ and $l_{\mathrm{a}}^{\mathrm{MFT}}=-\left(1+\underline{\delta}_{\mathrm{a}}\right)$ for $\mathrm{Cl}, \mathrm{Br}$, and I on (111) surfaces of fcc metals, as derived from published theoretical data [74] (see section 7 in the SI). Positive and negative values of $\underline{\delta}_{\mathrm{a}}$ classify systems according to their work function change (anomalous/normal) and are directly related to peak shifts $\frac{\mathrm{d}}{\mathrm{d} \log \left(c_{\mathrm{a}}\right)} P($ eq. $\sqrt{36})$ ) which we report on the right y-axis (Nernstian shift: $\frac{\mathrm{d}}{\mathrm{d} \log \left(\left(_{\mathrm{a}}\right)\right.} P=60 \mathrm{mV} /$ decade).

solution of $c_{\mathrm{a}}=1 \mathrm{M}$. Evidently, the CHE error $P-P^{\mathrm{CHE}}$ can easily vary in a range $\pm 0.2 \mathrm{~V}+$, which is far from negligible and on the same scale as inherent DFT inaccuracies. Furthermore, while the latter is often transferable (e.g. between substrates), Fig. 5 does not suggest this for the DL-induced shifts. However, a certain degree of correlation in the data points exists, which might enable a more accessible understanding in the future.

Note as well, that the magnitude and sign of peak shifts $\frac{\mathrm{d}}{\mathrm{d} C_{0}} P$ (eq. $\sqrt{39}$ ) follow the same behaviour of the CHE error and thus depend on the product of $\underline{\delta}_{\mathrm{a}}$ and $\Delta \Phi_{E}$. As a result, the magnitude and direction depends on how far the electrosorption peak is positioned left or right of the PZC, and whether the adsorbates induce a normal(anomalous) work function change $\left(\underline{\delta}_{\mathrm{a}} \stackrel{(>)}{<0}\right.$, cf. Fig. 5 ).

3.3.3. Peak shapes, $H$ and $W$ Above considerations with respect to the rescaled driving force due to non-zero $\underline{\delta}_{\mathrm{a}}$ indicates that $\underline{\delta}_{\mathrm{a}}$ should as well have an impact on the peak width. Indeed, the analysis of the Frumkin model suggests (see section 5 in the SI), that this behaviour is captured by the first term in eq. (34) that is proportional to the product of CHE width $W^{\mathrm{CHE}}$ and $\underline{\delta}_{\mathrm{a}}$. This term induces a larger(smaller) peak width than expected from the CHE results for systems with normal(anomalous) work function change in line with the reduced(increased) driving force considerations.

The second term in eq. (34) is always negative, representing thus a peak contraction. This is tentatively related to the fact that in a grand canonical setup, the DL potential drop adapts to compensate for adsorbate dipoles. The dipole-field interaction with the so-induced counter-oriented field is always "attractive" and always reduces the total energy irrespective of the sign of charge, dipole or DL field, thus explaining in sign and magnitude the second

+ Extreme values as $0.8 \mathrm{~V}$ for I on Pt are at very low potentials, and outside the stability window of water also in alkaline conditions. 


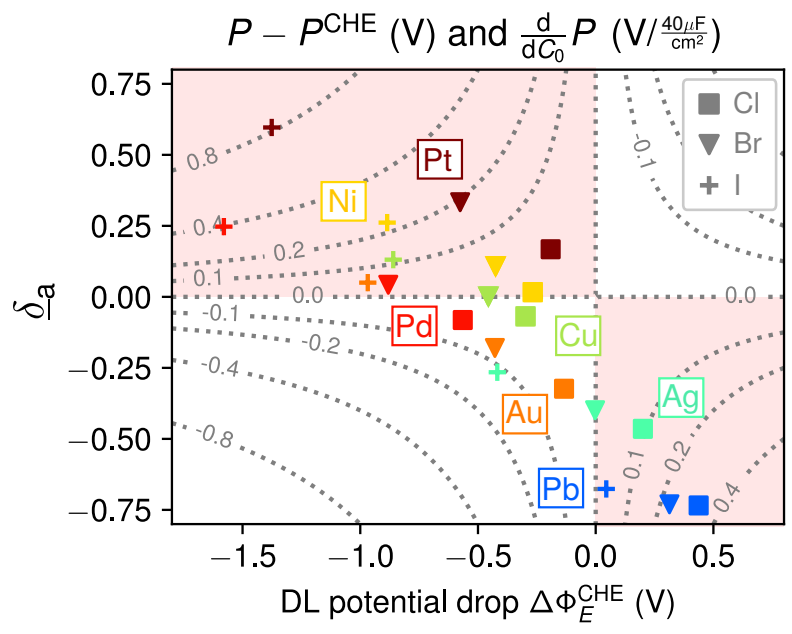

Figure 5. CHE peak accuracy $P-P^{\mathrm{CHE}}$ and peak shift as a change of the DL capacitance $\frac{\mathrm{d}}{\mathrm{d} C_{0}} P$. The contour lines are in logarithmic spacing with the indicated values in $\mathrm{V}$ for $P-P^{\mathrm{CHE}}$ and in $\mathrm{V} / \frac{40 \mu \mathrm{F}}{\mathrm{cm}^{2}}$ for $\frac{\mathrm{d}}{\mathrm{d} C_{0}} P$ (Note that both proxies are identical up to a multiplicative factor, cf. eqs. (30), (39)). Positive regions in the plot are colored in light red. Data points are obtained by combining experimental [76] and theoretical results [74] (see section 7 in the SI).

term in eq. (34) (see also SI). Note that these first two terms in eq. (34) are independent on $\Delta \Phi_{E}$ and thus represent a renormalization of the CHE results, as e.g. plotted in Fig. $3 \mathrm{a}$.

Finally the third term in eq. (34) takes the change of the adsorbate dipole with the coverage into account via the term $\frac{\mathrm{d}}{\mathrm{d} \bar{\theta}_{\mathrm{a}}} \underline{\delta}_{\mathrm{a}}$, which is directly related to the curvature of $\Phi_{0}^{\bar{\theta}_{\mathrm{a}}}$. It is only this third term in eq. (34) that varies with $\Delta \Phi_{E}$ and thus the only one that induces Non-Nernstian shape changes. This clarifies that alterations of the peak shape with changing experimental conditions, e.g. via $c_{\mathrm{a}}$, are mainly driven by the coverage-dependence of the adsorbate dipoles.

Similarly as for $\underline{\delta}_{\mathrm{a}}$, the sign of $\frac{\mathrm{d}}{\mathrm{d} \overline{\mathrm{a}}_{\mathrm{a}}} \delta_{\mathrm{a}}-$ which also occurs in the other shape-related proxies in tables 1 and 2 - depends on whether systems show a normal or an anomalous work function change and whether the magnitude of the adsorbate dipole increases or decreases with coverage. For systems with a normal work function change, $\frac{\mathrm{d}}{\mathrm{d} \bar{\theta}_{\mathrm{a}}} \underline{\delta}_{\mathrm{a}}<0$ when the magnitude of the adsorbate dipole increases with coverage as e.g. for halides on $\mathrm{Ag}(111)$. Respective alternative statements can be made for the 3 other possible scenarios thus allowing to explain a variety of non-trivial peak shape changes with changing experimental conditions.

For reasons of length, we will only take a closer look at eq. (41) in table 2, as it describes how peak heights can change with changing $C_{0}$. This is not only important for a better understanding of experiments, but also as it allows to estimate potential errors made in theoretical calculations with inaccurate DL capacitance $C_{0}$, e.g. in implicit solvation models.

Note that $M$ in eq. (41) is a unitless scaling factor where the numerical value of all contributing terms (eq. (42)) can be estimated in a straightforward way (e.g. the ratio between peak height $H$ and the baseline DL capacitance $C_{0}$ ). Furthermore, it is gratifying that all dependencies on $K$ have vanished, which makes us confident that the derived equation can be used to discuss any localized peak, without knowledge e.g. of the amount of coverage 
change that it encloses. All quantities have then to be interpreted as quantities at the respective interface composition. Note also, that eq. (41) suggests that the height of a CV peak changes exponentially with the capacitance, which indicates possible dramatic shape changes induced by moderate changes in $C_{0}$. Furthermore, the highest sensitivity to changes in $C_{0}$ is observed for high and narrow peaks. Equations (41) and (42) can be analysed for certain limiting cases:

(i) Case: $D_{\mathrm{a}}=\frac{\underline{\delta}_{\mathrm{a}}}{C_{0}} \rightarrow 0 ; \frac{\mathrm{d}}{\mathrm{d} \bar{\theta}_{\mathrm{a}}} D_{\mathrm{a}} \rightarrow 0$ (small adsorbate dipoles, non-zero $C_{0}$ ) Then $\frac{\mathrm{d}}{\mathrm{d} C_{0}} H \rightarrow 0$, consistent with the absence of capacitive charging effects.

(ii) Case: $C_{0} \rightarrow 0 ; D_{\mathrm{a}} \nrightarrow 0$ (small $C_{0}$, non-zero adsorbate dipoles) Then $\frac{\mathrm{d}}{\mathrm{d} C_{0}} H \nrightarrow 0$ as $M \nrightarrow 0$. Note that in this limit $M$ is largely independent on $C_{0}$, with the central term in eq. (42) vanishing.

(iii) Case: $D_{\mathrm{a}} \rightarrow 0 ; \frac{\mathrm{d}}{\mathrm{d} \bar{\theta}_{\mathrm{a}}} D_{\mathrm{a}} \nrightarrow 0$ (small overall dipole magnitude, however dipole magnitude still varies with coverage)

Then

$$
\frac{\mathrm{d}}{\mathrm{d} C_{0}} H \approx \sigma \frac{H^{2} \Delta \Phi_{E}}{\left|q_{\mathrm{a}}\right|} \frac{\mathrm{d}}{\mathrm{d} \bar{\theta}_{\mathrm{a}}} D_{\mathrm{a}} .
$$

Note that very narrow and high peaks (large $H$ ) studied away from the PZC (large $\left|\Delta \Phi_{E}\right|$ ) can thus be extremely sensitive to small changes in the DL capacitance $C_{0}$. In addition, the height/shape change with $C_{0}$ is expected to switch signs, whenever the system is studied below or above the PZC (sign of $\Delta \Phi_{E}$ ). These results clarify, that even in the case of small adsorbate dipoles $\left(D_{\mathrm{a}} \rightarrow 0\right)$, and thus naively expected small double layer effects, a non-vanishing coverage dependence of the adsorbate dipoles $\left(\frac{\mathrm{d}}{\mathrm{d} \bar{\theta}_{\mathrm{a}}} D_{\mathrm{a}} \neq 0\right)$ can lead to significant peak shape changes.

(iv) Case: Halides on $\operatorname{Ag}(111)$

Here, all terms in eq. 41 are of similar magnitude (theoretically), and thus the (theoretical) behaviour as a function of $C_{0}$ is extremely complicated and non-trivial, which we will not further elaborate here, but potentially come back to in subsequent work.

\section{Conclusions}

In this work we showed how all properties of thermodynamic $\mathrm{CV}$ peaks can in principle be mapped back to the coverage and potential dependence of the underlying fully grand canonical energetics. Note that eq. (15) represents in essence the first two terms in a possibly more complex Taylor expansion around the PZC of the interface energetics, with the difference between applied potential and the PZC $\left(\Phi_{E}-\Phi_{0}^{\bar{\theta}_{\mathrm{a}}}\right)$ as the relevant expansion variable. As a result, any possible further potential dependence (e.g. of the adsorbate dipoles, or the interfacial capacitance) would naturally induce additional and potentially higher order contributions, which can not be analysed analytically considering eq. (15). However, already at the present level of theory (quadratic equation in $\left.\left(\Phi_{E}-\Phi_{0}^{\bar{\theta}_{\mathrm{a}}}\right)\right)$ the results are not any more 
amenable to insightful analysis. On the other hand, when restricting ourselves to the case where the quadratic term is neglected ( $a=0$ in eq. (15)) a straightforward analytical analysis is possible, where only knowledge of the coverage-dependence of quantities evaluated at the PZC is necessary. The relevant quantities exhibit a straightforward interpretation e.g. via adsorbate dipoles and can be accessed directly e.g. from $a b$ initio calculations. This approximation corresponds to systems where $C_{0}^{\bar{\theta}_{a}}$ is largely coverage and potential independent. Note, that all mathematical terms that we referred to with "adsorbate dipole" are related fundamentally to coverage-induced work function changes and might also arise from other physical realities, e.g. a work function change due to the alteration of the interfacial water structure, which only changes the physical cause of these terms in the equations but not the derived results.

Using this and sensible additional approximations, as detailed in the SI, we were able to derive a list of proxies, that allow an insightful analysis and understanding of how ion concentrations and other electrolyte properties can lead to non-trivial changes in the observed $\mathrm{CV}$ peak positions and shapes. The successful test of these proxies on $\operatorname{Ag}(111)$ in halide containing solutions, where some of the assumptions made along the derivations are expected problematic, convinces us that they do indeed hold as well for other systems and provide valuable new information for an appropriate analysis of experimental results.

Among other things a markedly different behaviour for adsorbates with normal and anomalous work function is predicted. Furthermore, we have shown, that a variation in the capacitance $C_{0}$ can easily induce complex peak shifts and shape changes (cf. table 2). As a result, experimental $\mathrm{CV}$ measurement without controlled and known capacitance value $C_{0}$, which is often not reported, can be hardly analysed and understood in full completeness. In addition, the provided proxies allow us to pinpoint the main challenges of accurately predicting thermodynamic CVs via ab initio methods:

These are first and foremost the intrinsic inaccuracies of the adsorption energetics, as these values not only directly affect the predicted peak position but also directly impact the accuracy of the DL potential drop $\Delta \Phi_{E}$, and thereby any related quantity. Interestingly, however, such inaccuracies do not affect the dependencies on $\log \left(c_{\mathrm{a}}\right)$ (cf. table 2). The other relevant quantities are the PZC as well as its change with coverage and the interfacial capacitance. Evidently, also here, ab initio methods struggle, either due to overly simplified atomistic models (e.g. neglect of explicit interfacial water), and/or due to inherent inaccuracies of implicit solvent models.

While fully acknowledging these existing problems of theoretical modelling and not providing solutions to these, the present work provides important insight in how the described errors transfer to CHE+DL (fully grand canonical) results. In addition, the proxies allow error estimates for the more simple, but widely used, CHE energetics and thus clarifies cases where this method is problematic (cf. Fig. 5). In addition, we hope this work will help in the future to understand better the intricate details of thermodynamic CVs, e.g. their change with experimental conditions, and allow the reconstruction of atomistic properties of the interface that can be resolved and studied in a straightforward way via theoretical simulations. 


\section{Data availability statement}

All relevant data of this work is either compiled in the SI or available from the cited References.

\section{Acknowledgements}

The authors acknowledge financial support through the EuroTech Postdoc Programme which is co-funded by the European Commission under its framework programme Horizon 2020 and Grant Agreement number 754462 as well as support from the Deutsche Forschungsgemeinschaft (DFG, German Research Foundation) under Germany's Excellence Strategy - EXC 2089/1-390776260. This work was supported by a grant from the Gauss Centre for Supercomputing e.V. (www.gauss-centre.eu) by providing computing time through the John von Neumann Institute for Computing (NIC) on the GCS Supercomputer JUWELS [77] at Juelich Supercomputing Centre (JSC).

\section{References}

[1] Tiwari A, Heenen H H, Bjørnlund A S, Maagaard T, Cho E, Chorkendorff I, Kristoffersen H H, Chan K and Horch S 2020 Fingerprint voltammograms of copper single crystals under alkaline conditions: A fundamental mechanistic analysis J. Phys. Chem. Lett. 11 1450-5

[2] Ringe S, Morales-Guio C G, Chen L D, Fields M, Jaramillo T F, Hahn C and Chan K 2020 Double layer charging driven carbon dioxide adsorption limits the rate of electrochemical carbon dioxide reduction on Gold Nat. Commun. 1133

[3] Wang H, Thiele A and Pilon L 2013 Simulations of Cyclic Voltammetry for Electric Double Layers in Asymmetric Electrolytes: A Generalized Modified Poisson-Nernst-Planck Model J. Phys. Chem. C 117 18286-97

[4] Karlberg G S, Jaramillo T F, Skúlason E, Rossmeisl J, Bligaard T and Nørskov J K 2007 Cyclic Voltammograms for $\mathrm{H}$ on $\mathrm{Pt}(111)$ and $\mathrm{Pt}(100)$ from First Principles Phys. Rev. Lett. 99(12) 126101

[5] Asiri H A and Anderson A B 2013 Using Gibbs Energies to Calculate the Pt(111) Hupd Cyclic Voltammogram J. Phys. Chem. C 117 17509-13

[6] Chen J, Luo S, Liu Y and Chen S 2016 Theoretical Analysis of Electrochemical Formation and Phase Transition of Oxygenated Adsorbates on Pt(111) ACS Appl. Mater. Interfaces 8 20448-58

[7] McCrum I T and Janik M J 2016 pH and Alkali Cation Effects on the Pt Cyclic Voltammogram Explained Using Density Functional Theory J. Phys. Chem. C 120 457-71

[8] McCrum I T and Janik M J 2016 First Principles Simulations of Cyclic Voltammograms on Stepped Pt(553) and Pt(533) Electrode Surfaces ChemElectroChem 3 1609-17

[9] Kristoffersen H H, Vegge T and Hansen H A $2018 \mathrm{OH}$ formation and $\mathrm{H} 2$ adsorption at the liquid water-Pt(111) interface Chem. Sci. 9(34) 6912-21

[10] Bagger A, Arán-Ais R M, Halldin Stenlid J, Campos dos Santos E, Arnarson L, Degn Jensen K, EscuderoEscribano M, Roldan Cuenya B and Rossmeisl J 2019 Ab Initio Cyclic Voltammetry on Cu(111), $\mathrm{Cu}(100)$ and $\mathrm{Cu}(110)$ in Acidic, Neutral and Alkaline Solutions ChemPhysChem 20 3096-105

[11] Li Y and Janik M J 2019 Recent progress on first-principles simulations of voltammograms Curr. Op. Electrochem. $14124-132$

[12] Rossmeisl J, Jensen K D, Petersen A S, Arnarson L, Bagger A and Escudero-Escribano M 2020 Realistic Cyclic Voltammograms from Ab Initio Simulations in Alkaline and Acidic Electrolytes J. Phys. Chem. C 124 20055-65 
[13] Hörmann N G and Reuter K 2021 Thermodynamic Cyclic Voltammograms Based on Ab Initio Calculations: $\operatorname{Ag}(111)$ in Halide-Containing Solutions J. Chem. Theory Comput. 17 1782-94

[14] Vetter K J and Schultze J W 1972 Stromfluß bei Elektrosorptionsprozessen und Elektrosorptionswertigkeit $\gamma$ Ber. Bunsenges. Phys. Chem. 76 927-33

[15] Schmickler W 1988 The surface dipole moment of species adsorbed from a solution J. Electroanal. Chem. Interf. Electrochem. $24925-33$

[16] Hörmann N G, Marzari N and Reuter K 2020 Electrosorption at metal surfaces from first principles npj Comput. Mater. 6136

[17] Garlyyev B, Xue S, Watzele S, Scieszka D and Bandarenka A S 2018 Influence of the Nature of the Alkali Metal Cations on the Electrical Double-Layer Capacitance of Model Pt(111) and Au(111) Electrodes J. Phys. Chem. Lett. $91927-30$

[18] Ringe S, Clark E L, Resasco J, Walton A, Seger B, Bell A T and Chan K 2019 Understanding cation effects in electrochemical CO2 reduction Energy Environ. Sci. 12(10) 3001-14

[19] Nørskov J K, Rossmeisl J, Logadottir A, Lindqvist L, Kitchin J R, Bligaard T and Jonsson H 2004 Origin of the Overpotential for Oxygen Reduction at a Fuel-Cell Cathode J. Phys. Chem. B 108 17886-92

[20] Urushihara M, Chan K, Shi C and Nørskov J K 2015 Theoretical Study of EMIM+ Adsorption on Silver Electrode Surfaces J. Phys. Chem. C 119 20023-9

[21] Hansen M H, Jin C, Thygesen K S and Rossmeisl J 2016 Finite Bias Calculations to Model Interface Dipoles in Electrochemical Cells at the Atomic Scale J. Phys. Chem. C 120 13485-91

[22] Kastlunger G, Lindgren P and Peterson A A 2018 Controlled-Potential Simulation of Elementary Electrochemical Reactions: Proton Discharge on Metal Surfaces J. Phys. Chem. C 122 12771-81

[23] Hörmann N G, Guo Z, Ambrosio F, Andreussi O, Pasquarello A and Marzari N 2019 Absolute band alignment at semiconductor-water interfaces using explicit and implicit descriptions for liquid water npj Comput. Mater. 5100

[24] Senftle T P, Hong S, Islam M M, Kylasa S B, Zheng Y, Shin Y K, Junkermeier C, Engel-Herbert R, Janik M J, Aktulga H M, Verstraelen T, Grama A and van Duin A C T 2016 The ReaxFF reactive force-field: development, applications and future directions. npj Comput. Mater. 215011

[25] Bonnet N, Morishita T, Sugino O and Otani M 2012 First-Principles Molecular Dynamics at a Constant Electrode Potential Phys. Rev. Lett. 109(26) 266101

[26] Bouzid A and Pasquarello A 2018 Atomic-Scale Simulation of Electrochemical Processes at Electrode/Water Interfaces under Referenced Bias Potential J. Phys. Chem. Lett. 9 1880-4

[27] Surendralal S, Todorova M, Finnis M W and Neugebauer J 2018 First-principles approach to model electrochemical reactions: Understanding the fundamental mechanisms behind mg corrosion Phys. Rev. Lett. 120(24) 246801

[28] Hansen M H and Rossmeisl J 2016 pH in Grand Canonical Statistics of an Electrochemical Interface J. Phys. Chem. C 120 29135-43

[29] Le J B, Fan Q Y, Li J Q and Cheng J 2020 Molecular origin of negative component of Helmholtz capacitance at electrified $\mathrm{Pt}(111) /$ water interface $S c i . A d v .6$

[30] ENVIRON package Http://www.quantum-environment.org

[31] Andreussi O, Dabo I and Marzari N 2012 Revised self-consistent continuum solvation in electronicstructure calculations J. Chem. Phys. 136064102

[32] Mathew K, Sundararaman R, Letchworth-Weaver K, Arias T A and Hennig R G 2014 Implicit solvation model for density-functional study of nanocrystal surfaces and reaction pathways J. Chem. Phys. 140 084106

[33] Held A and Walter M 2014 Simplified continuum solvent model with a smooth cavity based on volumetric data J. Chem. Phys. 141174108

[34] Sundararaman R, Letchworth-Weaver K, Schwarz K A, Gunceler D, Ozhabes Y and Arias T 2017 JDFTx: Software for joint density-functional theory SoftwareX $6278-284$

[35] Letchworth-Weaver K and Arias T A 2012 Joint density functional theory of the electrode-electrolyte interface: Application to fixed electrode potentials, interfacial capacitances, and potentials of zero charge Phys. Rev. B 86(7) 075140 
[36] Bonnet N and Marzari N 2013 First-Principles Prediction of the Equilibrium Shape of Nanoparticles Under Realistic Electrochemical Conditions Phys. Rev. Lett. 110(8) 086104

[37] Bonnet N, Dabo I and Marzari N 2014 Chemisorbed Molecules under Potential Bias: Detailed Insights from First-Principles Vibrational Spectroscopies Electrochim. Acta 121210 - 214

[38] Lespes N and Filhol J S 2015 Using Implicit Solvent in Ab Initio Electrochemical Modeling: Investigating $\mathrm{Li}+/ \mathrm{Li}$ Electrochemistry at a Li/Solvent Interface J. Chem. Theory Comput. 11 3375-82

[39] Fisicaro G, Genovese L, Andreussi O, Marzari N and Goedecker S 2016 A generalized Poisson and Poisson-Boltzmann solver for electrostatic environments J. Chem. Phys. 144014103

[40] Fisicaro G, Genovese L, Andreussi O, Mandal S, Nair N N, Marzari N and Goedecker S 2017 Soft-Sphere Continuum Solvation in Electronic-Structure Calculations J. Chem. Theory Comput. 13 3829-45

[41] Ringe S, Oberhofer H and Reuter K 2017 Transferable ionic parameters for first-principles PoissonBoltzmann solvation calculations: Neutral solutes in aqueous monovalent salt solutions J. Chem. Phys. 146134103

[42] Sundararaman R and Schwarz K 2017 Evaluating continuum solvation models for the electrode-electrolyte interface: Challenges and strategies for improvement J. Chem. Phys. 146084111

[43] Sundararaman R, Goddard W A and Arias T A 2017 Grand canonical electronic density-functional theory: Algorithms and applications to electrochemistry J. Chem. Phys. 146114104

[44] Sundararaman R, Figueiredo M C, Koper M T M and Schwarz K A 2017 Electrochemical Capacitance of CO-Terminated Pt(111) Dominated by the CO-Solvent Gap J. Phys. Chem. Lett. 8 5344-8

[45] Ping Y, Nielsen R J and Goddard W A 2017 The Reaction Mechanism with Free Energy Barriers at Constant Potentials for the Oxygen Evolution Reaction at the IrO2 (110) Surface J. Am. Chem. Soc. $139149-55$

[46] Huang J, Hörmann N, Oveisi E, Loiudice A, De Gregorio G L, Andreussi O, Marzari N and Buonsanti R 2018 Potential-induced nanoclustering of metallic catalysts during electrochemical $\mathrm{CO} 2$ reduction Nat. Commun. 93117

[47] Zhang H, Goddard W A, Lu Q and Cheng M J 2018 The importance of grand-canonical quantum mechanical methods to describe the effect of electrode potential on the stability of intermediates involved in both electrochemical CO2 reduction and hydrogen evolution Phys. Chem. Chem. Phys. 20(4) 2549-57

[48] Nattino F, Truscott M, Marzari N and Andreussi O 2019 Continuum models of the electrochemical diffuse layer in electronic-structure calculations J. Chem. Phys. 150041722

[49] Andreussi O, Hörmann N G, Nattino F, Fisicaro G, Goedecker S and Marzari N 2019 Solvent-Aware Interfaces in Continuum Solvation J. Chem. Theory Comput. 15 1996-2009

[50] Hörmann N G, Andreussi O and Marzari N 2019 Grand canonical simulations of electrochemical interfaces in implicit solvation models J. Chem. Phys. 150041730

[51] Gauthier J A, Ringe S, Dickens C F, Garza A J, Bell A T, Head-Gordon M, Nørskov J K and Chan K 2019 Challenges in Modeling Electrochemical Reaction Energetics with Polarizable Continuum Models $A C S$ Catal. 9 920-31

[52] Mitchell S, Brown G and Rikvold P 2000 Dynamics of Br electrosorption on single-crystal $\operatorname{Ag}(100)$ : a computational study J. Electroanal. Chem. $49368-74$

[53] Mitchell S, Brown G and Rikvold P 2001 Static and dynamic Monte Carlo simulations of $\mathrm{Br}$ electrodeposition on $\operatorname{Ag}(100)$ Surf. Sci. 471125 - 142

[54] Weitzner S E and Dabo I 2017 Quantum-continuum simulation of underpotential deposition at electrified metal-solution interfaces npj Comput. Mater. 31

[55] Weitzner S E and Dabo I 2017 Voltage-dependent cluster expansion for electrified solid-liquid interfaces: Application to the electrochemical deposition of transition metals Phys. Rev. B 96(20) 205134

[56] Ambrosio F, Wiktor J and Pasquarello A 2018 pH-Dependent Surface Chemistry from First Principles: Application to the BiVO4(010)-Water Interface ACS Appl. Mat. E Interf. 10 10011-21

[57] Peterson A A, Abild-Pedersen F, Studt F, Rossmeisl J and Nørskov J K 2010 "how copper catalyzes the electroreduction of carbon dioxide into hydrocarbon fuels" Energy Environ. Sci. 3(9) 1311-5

[58] Rossmeisl J, Nørskov J K, Taylor C D, Janik M J and Neurock M 2006 Calculated Phase Diagrams for the Electrochemical Oxidation and Reduction of Water over Pt(111) J. Phys. Chem. B 110 21833-9 
[59] Mamatkulov M and Filhol J S 2011 An abinitio study of electrochemical vs. electromechanical properties: the case of CO adsorbed on a Pt(111) surface Phys. Chem. Chem. Phys. 13(17) 7675-84

[60] Filhol J S and Doublet M L 2013 An ab initio study of surface electrochemical disproportionation: The case of a water monolayer adsorbed on a Pd(111) surface Catal. Today 20287 - 97

[61] Steinmann S N, Michel C, Schwiedernoch R and Sautet P 2015 Impacts of electrode potentials and solvents on the electroreduction of CO2: a comparison of theoretical approaches Phys. Chem. Chem. Phys. 17(21) 13949-63

[62] Gauthier J A, Dickens C F, Ringe S and Chan K 2019 Practical Considerations for Continuum Models Applied to Surface Electrochemistry ChemPhysChem 20 3074-80

[63] Gauthier J A, Dickens C F, Heenen H H, Vijay S, Ringe S and Chan K 2019 Unified Approach to Implicit and Explicit Solvent Simulations of Electrochemical Reaction Energetics J. Chem. Theory Comput. 15 6895-906

[64] Lozovoi A Y and Alavi A 2003 Reconstruction of charged surfaces: General trends and a case study of $\mathrm{Pt}(110)$ and $\mathrm{Au}(110)$ Phys. Rev. B 68(24) 245416

[65] Reuter K 2016 Ab Initio Thermodynamics and First-Principles Microkinetics for Surface Catalysis Catal. Lett. 146 541-63

[66] Hörmann N, Jäckle M, Gossenberger F, Roman T, Forster-Tonigold K, Naderian M, Sakong S and Groß A 2015 Some challenges in the first-principles modeling of structures and processes in electrochemical energy storage and transfer J. Power Sources $275531-538$

[67] Gossenberger F, Roman T and Groß A 2015 Equilibrium coverage of halides on metal electrodes Surf. Sci. $63117-22$

[68] Andreussi O, Nattino F and Hörmann N G 2020 Chapter: Continuum Embedding Models for Electrolyte Solutions in First-Principles Simulations of Electrochemistry Atomic-Scale Modelling of Electrochemical Systems ed Melander M, Laurila T and Laasonen K (Copyright (IN PRESS) John Wiley and Sons Ltd. Please contact publishers for details)

[69] Zunger A, Wei S H, Ferreira L G and Bernard J E 1990 Special quasirandom structures Phys. Rev. Lett. 65(3) 353-6

[70] van de Walle A 2009 Multicomponent multisublattice alloys, nonconfigurational entropy and other additions to the Alloy Theoretic Automated Toolkit Calphad 33266 - 278 tools for Computational Thermodynamics

[71] Hörmann N G and Groß A 2019 Phase field parameters for battery compounds from first-principles calculations Phys. Rev. Mater. 3(5) 055401

[72] Foresti M L, Innocenti M, Forni F and Guidelli R 1998 Electrosorption Valency and Partial Charge Transfer in Halide and Sulfide Adsorption on Ag(111) Langmuir 14 7008-16

[73] Roman T and Groß A 2013 Periodic Density-Functional Calculations on Work-Function Change Induced by Adsorption of Halogens on $\mathrm{Cu}(111)$ Phys. Rev. Lett. 110(15) 156804

[74] Roman, Tanglaw and Gossenberger, Florian and Forster-Tonigold, Katrin and Groß, Axel 2014 Halide adsorption on close-packed metal electrodes Phys. Chem. Chem. Phys. 16(27) 13630-4

[75] Gossenberger F, Roman T, Forster-Tonigold K and Groß A 2014 Change of the work function of platinum electrodes induced by halide adsorption Beilstein J. Nanotechnol. 5 152-61

[76] White R E, Bockris J O and Conway B E (eds) 1999 Modern Aspects of Electrochemistry 1st ed (Modern Aspects of Electrochemistry vol 33) (Springer US)

[77] Jülich Supercomputing Centre 2019 JUWELS: Modular Tier-0/1 Supercomputer at the Jülich Supercomputing Centre Journal of large-scale research facilities $\mathbf{5}$ 